\title{
Experimental Mueller matrix polarimetry with full Poincaré beams and a CCD camera.
}

\author{
Juan Carlos Suárez-Bermejo ${ }^{1}$, J. Carlos González de Sande ${ }^{2}$, Massimo Santarsiero $^{3}$ and Gemma Piquero ${ }^{4}$ \\ ${ }^{1}$ Universidad Politécnica de Madrid, Materials Science Department, Av. de la Memoria 4, 28040 Madrid, Spain \\ ${ }^{2}$ Universidad Politécnica de Madrid, ETSIS de Telecomunicación, Campus Sur, 28031 Madrid, Spain \\ ${ }^{3}$ Università Roma Tre, Dipartimento di Ingegneria, V. Volterra 62, Rome 00146, Italy \\ ${ }^{4}$ Universidad Complutense de Madrid, Departamento de Óptica, 28040 Madrid, Spain
}

\begin{abstract}
Recently, the use of full Poincaré beams for extracting the Mueller matrix of a sample has been proposed. These beams present all possible polarization states across their transverse section. By placing a CCD camera behind a simple polarization analyzer formed by a quarter wave phase plate and a linear polarizer, a polarization map of the beam cross section can be obtained. This polarization map is modified when a sample is inserted before the polarization state analyzer. Comparison of these two polarization maps allows to obtain the Mueller matrix of the sample. An overdetermined system of linear equations (thousands of equations) can be written from this comparison. Standard mathematical methods are used to find optimum solution of this overdetermined system of equations. Some experimental results will be presented to check the performance of the proposed polarimetric method.
\end{abstract}

\section{Introduction}

The use of non-uniformly totally polarized (NUTP) beams $[1,2]$ has been proposed as parallel polarization states generator (PSG) for Mueller polarimetry [3,4]. Experimental measurement of Mueller matrices has been done by using azimuthally [5] or spirally [6] polarized beams. In these cases, only linear polarization states are present across the beam section, so that only a partial Mueller matrix can be obtained, but this is enough for recovering the whole matrix for homogeneous and deterministic samples [5-8]. If four independent states of totally polarized light are present across the NUTP beam section [9], the complete Mueller matrix can be measured for any sample $[10,11]$. The so-called full Poincaré beams (FPBs) contain all possible polarization states across its transverse section $[9,12,13]$ and are candidates to be used as parallel PSGs.

Two alternative schemes have been proposed to obtain the Mueller matrix of a sample with FPBs. One is based on the use of a commercial polarimeter for measuring the state of polarization at four different points across the beam transverse section, before and after the sample under study. The inversion of the polarimetric relation gives the Mueller matrix of the sample. This procedure has been carried out experimentally and good results has been obtained [10]. The second method is based on the use of a CCD camera and a polarization state analyser [14]. Intensity measurements are made behind a polarizer and a quarter wave phase plate that are placed at least in four different configurations. As the input beam is a FPB, the change in the state of polarization at each point of its transverse section can be measured and a large data set can be obtained. Numerical simulations using different strategies to obtain the Mueller matrix of the sample from these data have been recently presented. These strategies are the denoted as center-point strategy and nearestneighbor strategy [14].

In this work, a different approach to the problem is proposed, which will be called the Moore-Penrose (M-P) pseudo-inverse strategy. To obtain information about the sample we will use not only four, but a larger number of points across the beam section and apply the M-P pseudoinverse strategy to simulated images affected by Gaussian noise. The obtained results will be compared to those provided by previous procedures, in order to verify which one gives the most accurate results. The noise caused by inaccuracies in the measurement of the intensity captured by each pixel of the CCD camera will be carefully analysed. The theoretical results will be compared with the results obtained from experimental images in a particular case.

In Section 2, the general procedure for FPB Mueller matrix polarimetry is sketched. Numerical simulations are presented in Section 3 and some of them are compared to experimental results in Section 4. Finally, concluding remarks are outlined.

\section{Mueller full Poincaré polarimetry}

The scheme followed to acquire the raw data is shown in Fig. 1. A particular FPB is synthesized by means of a calcite crystal $[1,9]$ and is used as a PSG. The polarization pattern of this beam is characterized by means of a

\footnotetext{
* Corresponding author: juancarlos.suarez@upm.es
} 
polarization state analyser (PSA), which consists of a polarizer and a quarter wave phase plate. The intensity profile for at least four different configurations of the PSA is measured by a CCD camera [see Fig. 1 a)]. From these intensity measurements, the Stokes parameters at each point of the input beam can be determined. Afterwards, a known sample is placed before the PSA and the intensity map acquisition is repeated [see Fig. 1 b)]. From this second set of intensity maps, the Stokes parameters at each point of the transverse section of the output beam can be obtained.

a)

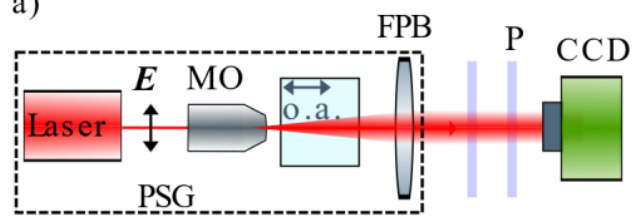

QWP

b)

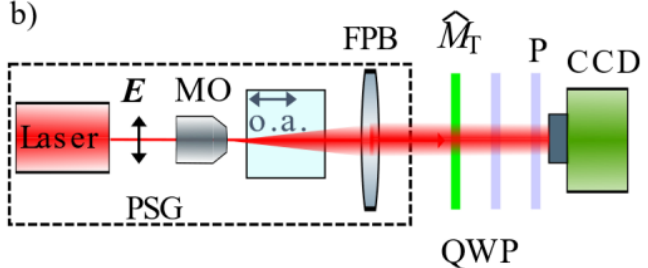

Fig. 1. Experimental scheme for Mueller FPB Polarimetry without (a) and with (b) a sample. E: MO: objective of microscope, o.a.: optical axis of a calcite crystal; L: lens QWP: quarter wave phase plate; P: polarizer.

The Stokes parameters of the input beam and those of the output beam are related by the Mueller matrix, $\widehat{M}_{S}$, of the sample by

$$
\vec{S}^{\text {out }}=\widehat{M}_{S} \vec{S}^{\text {in }} .
$$

We apply this relation to a wide area of the beam transverse section where the Poincaré sphere is mapped at least twice [9], so that an overdetermined system of equations is obtained. This system is inverted by means of the Moore-Penrose pseudo-inverse [15] to obtain the Mueller matrix of the sample.

An important source of error is the inaccuracy in the intensity measurements at each pixel of the CCD camera. Comparison of several images acquired under the same conditions has been performed to evaluate such source of error [16]. From this comparison, it has been deduced that the standard deviation of the measured intensity in each pixel of the CCD camera is about or below 0.01 times the mean value of the intensity [16].

\section{Numerical simulations}

Simulations for four different standard samples have been made: air (no sample introduced in the polarimetric system), a linear polarizer with horizontal transmission axis (LP0) and with its transmission axis at $60^{\circ}$ with respect to the horizontal (LP60), and a quarter wave phase (QWP) retarder with its fast axis along the horizontal direction.
Theoretical images of CCD power distribution for six specific configurations [14] of the PSA are generated using a specifically dedicated code in MATLAB. Input images are independently generated and then passed through a function that adds Gaussian noise (characterized by the standard deviation $\varepsilon$ of its probability density function). Input Stokes vectors for the four sample are derived from these noisy images. Regarding the output images, theoretical input Stokes vectors (without noise) are modified using the ideal Mueller matrix of each sample. These output Stokes vectors are used to obtain the output intensity images in the six considered states after the beam has passed through the sample. The power images are then sent to the modulus for adding Gaussian noise (randomly generated and, in consequence, independent from the noise in the input images). The Stokes vectors of the output beams at each pixel are then reconstructed from the output noisy images.

By using the noisy input and output sets of Stokes vectors, the Mueller matrix of the sample can be calculated by means of Eq. (1). This process is repeated many times. Then the difference between the theoretical Mueller matrix and those obtained from the simulations can be calculated. To give a measure of these differences with a sole number, the root mean square of such difference is calculated as

$$
\operatorname{rms}\left(\widehat{M}_{S}-\widehat{M}_{T}\right)=\frac{1}{4} \sqrt{\sum_{i, j=0}^{3}\left(m_{i j}^{S}-m_{i j}^{T}\right)^{2}},
$$

where $m_{i j}^{S}$ are the values of the Mueller matrix elements obtained from the simulation and $m_{i j}^{T}$ are the theoretical ones.

To obtain a quantitative estimate of the errors in the determination of Mueller matrix for a considered sample, three different strategies have been followed. In first place (center-point strategy), four points have been selected in such a way that their polarization states correspond to the vertices of a tetrahedron inscribed in the Poincare sphere. Using the same points for the input and the output, and obtaining the Stokes vectors at these specific locations, it is possible to obtain the Mueller matrix of the sample including the noise in the simulated measurements. The process can be repeated up to 15 realizations maintaining one of the vertices fixed and rotating the other three around the axis $s_{1}$. Afterwards, it is possible to obtain an average Mueller matrix from all the realizations and compare every individual element with the theoretical matrix to have an estimation of the errors for every value of the parameter $\varepsilon$.

In the second approach (nearest-neighbor strategy) not only four points are selected in every realization to calculate the Mueller matrix inverting Eq. 1, but a wider set of points is used, all located around each of the center points considered in the previous case. A total of $N \times N$ nearest neighbor pixels are considered around every central point. Mueller matrices are obtained for all the possible four-point sets (input and output) that can be chosen from the $4 \times N^{2}$ pixels included in the analysis. An average value is then computed from all the valid cases (condition number of the matrix below 20), for the 
15 analysed orientations of the tetrahedron in the Poincaré sphere.

Finally, the third strategy (Moore-Penrose pseudoinverse) uses all points included in a circle around the center of the images, up to a certain radius, to construct an overdetermined system of linear equations that gives an estimate of the 16 elements of a Mueller matrix with the minimum mean square error value. The radius of the considered circle must be great enough to contain the center part of the beam where all possible polarization states can be found twice $[9,10]$. Taking into account the CCD camera characteristics, this radius can be up to 600 pixels. The state of polarization, and hence the Stokes vector varies from pixel to pixel. Then, the calculations involve the individual values of around $1.13 \times 10^{6}$ pixels for every realization.

Gaussian noise has been introduced independently for every realization with a value for parameter $\varepsilon=0.01$, which is comparable to the standard deviation of the intensity measured values in each pixel of the CCD camera used in the experiment [16]. Figure 2 shows the root mean square of the difference for each realization of this simulation. In total, 500 realizations of the simulation have been done assuming the sample is air. It is found that the root mean square of the difference is around $1.14 \times 10^{-3}$, which is a quite low value [17]. It can be observed in Fig. 2 that the dispersion of these root mean squares is very small.

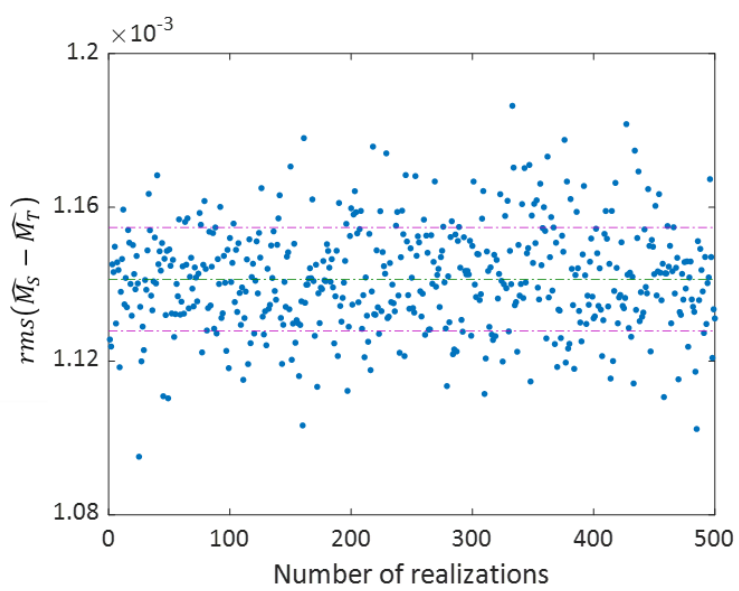

Fig. 2. Differences of rms (Eq. (2)) of air as a sample using 500 realizations of a numerical simulation, utilizing the MoorePenrose pseudo-inverse strategy $(\varepsilon=0.01)$.

The simulations have been repeated for three additional samples: a quarter wave plate with its fast axis along the horizontal direction, a linear polarizer with its transmission axis along the horizontal direction, and the same polarizer with its transmission axis forming a $60^{\circ}$ angle relative to positive horizontal direction. Figure 3 summarized the results obtained from the 500 realizations of the simulation for the four samples that have been considered. Fig. 3 shows the average of the 500 computed root mean squares and their standard deviation for every sample.

An interesting issue is to determine if the differences of the sixteen elements of the Mueller matrix are evenly distributed or if there are some elements in the Mueller matrix that are prone to present higher errors than the mean.

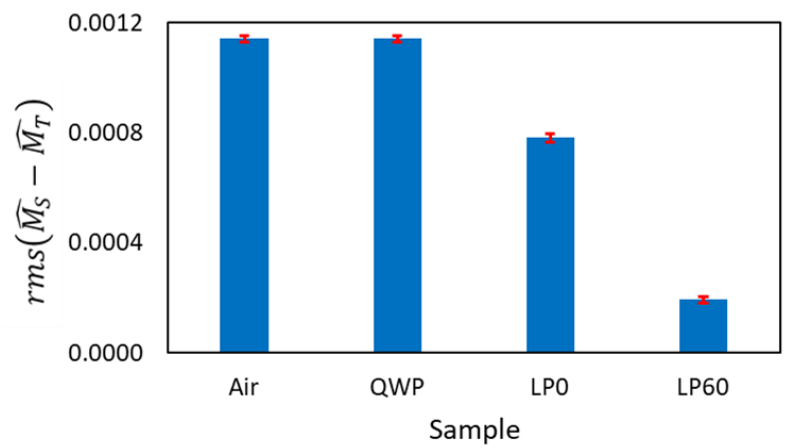

Fig. 3. Mean values and standard deviations of the root squared differences between the Mueller matrices obtained from numerical simulations and the ideal ones. For four samples: air, a linear polarizer with horizontal transmission axis (LP0) and with its transmission axis at $60^{\circ}$ with respect to the horizontal (LP60), and a quarter wave phase (QWP) retarder with its fast axis along the horizontal direction.

To elucidate this question, the differences of each individual element between the theoretical Mueller matrix and the one obtained for each simulation have been evaluated. After that, they have been represented as a set of dots in the vertical of the matrix element indexes. Figure 4 shows this representation for the case of air and taking added Gaussian noise with standard deviation $\varepsilon=0.02$. Again, 500 realizations of the simulation have been done considering all the pixels around the center of the images up to a radius of 600 pixels. It can be observed that the absolute value of all differences are below $5 \times 10^{-3}$, except for one element. The biggest difference is found for $m_{22}$ element for which the average value of the difference is $14 \times 10^{-3}$.

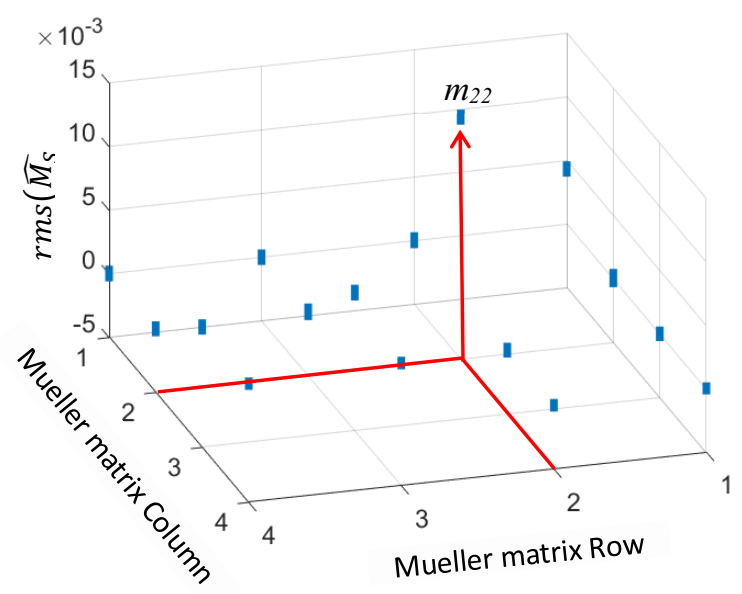

Fig. 4. Individual values of the differences of the $4 \times 4$ elements between Mueller matrix obtained for each realization of the simulation and the theoretical one. The case of air for 500 realizations of the simulation with a standard deviation of the added Gaussian noise $\varepsilon=0.02$ is shown.

\section{Experimental results}

As an example, just for comparative purposes, the measurements in air are shown here. The results for the 
rest of the samples will be published elsewhere. The experimental setup for generating the FPB can be found in ref. [9]. The obtained FPB is expanded to cover most of the CCD camera surface. Two different sets of imaged for six different configurations of the PSA [14] have been acquired. From these two sets of images, the air Mueller matrix has been obtained following the M-P pseudoinverse strategy. The root mean square value of the difference between the measured Mueller matrix and the identity matrix is $4 \times 10^{-3}$. This result is presented as a red horizontal line in Fig. 5. This is compared to the rms levels found from simulations for the three described strategies when a different level of added noise is considered. In the numerical simulations, no matter what noise level is added to the images, the differences with the ideal Mueller matrix of the sample are lower when the Moore-Penrose pseudo-inverse strategy is employed to compute the matrix, as compared with the center-point and the nearest-neighbor strategies (see Fig. 5). However, the computational cost is higher for the calculations using the pseudo-inverse because it is necessary to solve a system of linear equations involving $1.13 \times 10^{6}$ pixels in the images.

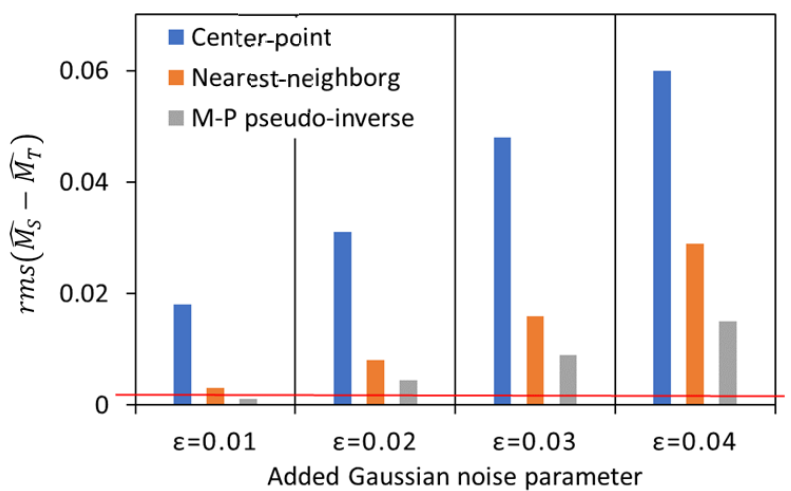

Fig. 5. Errors estimated in the numerical simulations for air, using three different strategies to compute the Mueller matrices, and experimental results for the sample.

It can be observed that the experimental result (red line in Fig. 5) is somewhere between the rms values obtained when the Moore-Penrose pseudo-inverse strategy is used in simulations where the standard deviation of the added Gaussian noise is 0.01 and 0.02 . This level of Gaussian noise is in good agreement with the independently determined noise in the images obtained with our CCD camera and polarimetric system [16].

\section{Conclusions}

Full Poincaré Mueller Polarimetry can be carried out using different setups. Here a PSA consisting of a polarizer, a quarter wave phase plate and a CCD camera is proposed. With this setup three different strategies for processing the data from the camera can be applied. Two of them (center-point strategy and nearest-neighbor strategy) have already been proposed in a previous work [14]. Here a new strategy (Moore-Penrose pseudo-inverse strategy) is applied to the simulated imagen with added noise for different samples and compared with the previous results. Finally, for a particular sample the numerical results have also been compared with the results when the experimental images are considered.

\section{Acknowledgements}

This work has been partially supported by Spanish Ministerio de Economía y Competitividad under project PID2019-104268GB-C21.

\section{References}

1. G. Piquero and J. Vargas-Balbuena, Eur. J. Phys. 25, 793 (2004).

2. G. Piquero. R. Martínez-Herrero, J.C.G. de Sande, M. Santarsiero, JOSA A 37, 591 (2020).

3. S. Tripathi, K.C. Toussaint KC. Opt. Express, 17, 21396 (2009).

4. F. Kenny, O. Rodríguez, D. Lara, C. Dainty, Frontiers in Optics 2011/Laser Science XXVII. Optical Society of America; (2011).

5. J.C.G. de Sande, G. Piquero, and M. Santarsiero, Opt. Commun. 410, 961 (2018).

6. J.C.G. de Sande, M. Santarsiero, and G. Piquero, Opt. Laser Eng. 91, 97 (2017).

7. R.A. Chipman, Polarimetry, in Handbook of Optics, ed, M. Bass, (McGraw-Hill Companies, 2010).

8. M. Swami, H. Patel, P. Gupta, Opt. Commun. 286, 18 (2013).

9. G. Piquero. L. Monroy, M. Santarsiero, M. Alonzo, J.C. de Sande, J. Opt. 20, 065602 (2018).

10. J. C. Suárez-Bermejo, J.C.G. de Sande, M. Santarsiero, G. Piquero, Opt. Laser Eng., 122, 134 (2019).

11. J.C. Suárez-Bermejo, J.C.G. de Sande, M. Santarsiero, G. Piquero, 2019-Progress In Electromagnetics Research Symposium, (PIERSRome) 2019.

12. A.M. Beckley, T.G. Brown, and M. A. Alonso, Opt. Express, 18, 10777 (2010).

13. E.J. Galvez, S. Khadka, W.H. Schubert, and S. Nomoto, Appl. Opt. 51, 2925 (2012).

14. J. C. Suárez-Bermejo, J.C.G. De Sande, M. Santarsiero, G. Piquero, Sensors \& Transducers 247 (8), 18-24 (2020).

15. A. Ben-Israel, T.N.E. Greville, Generalized inverses: Theory and applications (2nd ed.). New York, NY: Springer. (2003).

16. J. C. Suárez-Bermejo, J.C.G. de Sande, M. Santarsiero, G. Piquero, Advances in Optics: Reviews Book Series, Vol. 5 (2021).

17. J. del Hoyo, L.M. Sanchez-Brea, J.A. GomezPedrero Opt. Laser Eng., 132, 106112 (2020). 